\title{
Strain-specific impact of PsaR of Streptococcus pneumoniae on global gene expression and virulence
}

\begin{abstract}
Correspondence
Peter W. M. Hermans

P.Hermans@cukz.umcn.nl
\end{abstract}

Received 20 October 2008

Revised 16 January 2009

Accepted 2 February 2009

\author{
Wouter T. Hendriksen, ${ }^{1} \dagger$ Hester J. Bootsma, ${ }^{2}$ Angela van Diepen, ${ }^{2}$ \\ Silvia Estevão, ${ }^{1}$ Oscar P. Kuipers, ${ }^{3}$ Ronald de Groot ${ }^{2}$ \\ and Peter W. M. Hermans ${ }^{2}$
${ }^{1}$ Department of Pediatrics, Erasmus MC-Sophia Children's Hospital, 3000 DR Rotterdam, The Netherlands
${ }^{2}$ Laboratory of Pediatric Infectious Diseases, Radboud University Nijmegen Medical Centre, 6500 HB Nijmegen, The Netherlands
${ }^{3}$ Department of Molecular Genetics, University of Groningen, Groningen Biomolecular Sciences and Biotechnology Institute, PO Box 14, 9750 AA Haren, The Netherlands

\begin{abstract}
Previous studies have indicated that PsaR of Streptococcus pneumoniae is a manganesedependent regulator, negatively affecting the expression of at least seven genes. Here, we extended these observations by transcriptome and proteome analysis of $p s a R$ mutants in strains D39 and TIGR4. The microarray analysis identified three shared PsaR targets: the psa operon, $p c p A$ and prtA. In addition, we found 31 genes to be regulated by PsaR in D39 only, most strikingly a cellobiose-specific phosphotransferase system (PTS) and a putative bacteriocin operon (sp0142-sp0146). In TIGR4, 14 PsaR gene targets were detected, with the rIrA pathogenicity islet being the most pronounced. Proteomics confirmed most of the shared gene targets. To examine the contribution of PsaR to pneumococcal virulence, we compared D39 and TIGR4 wild-type (wt) and psaR mutants in three murine infection models. During colonization, no clear effect was observed of the psaR mutation in either D39 or TIGR4. In the pneumonia model, small but significant differences were observed in the lungs of mice infected with either D39wt or $\Delta p s a R$ : D39 $\Delta p s a R$ had an initial advantage in survival in the lungs. Conversely, TIGR4 $\Delta p s a R-$ infected mice had significantly lower bacterial loads at $24 \mathrm{~h}$ only. Finally, during experimental bacteraemia, D39 $\Delta$ psaR-infected mice had significantly lower bacterial loads in the bloodstream than wt-infected mice for the first $24 \mathrm{~h}$ of infection. TIGR4 $\Delta p s a R$ showed attenuation at $36 \mathrm{~h}$ only. In conclusion, our results show that PsaR of D39 and TIGR4 has a strain-specific role in global gene expression and in the development of bacteraemia in mice.
\end{abstract}

\section{INTRODUCTION}

Streptococcus pneumoniae encounters different environments during its life cycle: in most cases it inhabits the human nasopharynx, where it resides asymptomatically, but it can also spread through the body, causing severe infections (Bogaert et al., 2004). What exactly triggers the pneumococcus to cause infections at these sites in the body is poorly understood. In response to environmental

tPresent address: Section Molecular and Developmental Genetics, Institute of Biology, Leiden University, The Netherlands.

Abbreviations: CSP, competence-stimulating pepide; PTS, phosphotransferase system; SILAC, stable isotope labelling in cell culture.

The GEO series accession number for the microarray data associated with this paper is GSE 13505. changes, the transcriptional programme is likely to change, which is considered to be niche-specific and can result in expression of distinct virulence factors.

Manganese ions $\left(\mathrm{Mn}^{2+}\right)$ are important for bacterial life, for instance serving as cofactors for metalloenzymes. The function of these metalloenzymes is widespread among bacterial cellular processes, such as glycolysis, gluconeogenesis and oxidative stress defence (Jakubovics \& Jenkinson, 2001). In the pneumococcus, involvement of $\mathrm{Mn}^{2+}$ in competence has also been described (Dintilhac et al., 1997), but most studies have focused on its involvement in oxidative stress (Ibrahim et al., 2005; Johnston et al., 2004; McAllister et al., 2004; McCluskey et al., 2004; Paterson et al., 2006; Tseng et al., 2002). In the latter process, $\mathrm{Mn}^{2+}$ serves as a cofactor for superoxide 
dismutase, an important enzyme that provides defence against superoxide radicals (Archibald \& Fridovich, 1981). As the concentration of manganese is much higher in saliva than in blood plasma (Chicharro et al., 1999), fluctuations in the amount of this trace element might serve as a trigger for expression of certain virulence factors.

Various transcriptional regulators have been described for the pneumococcus (Hava et al., 2003b) and several largescale mutagenesis studies have identified a role in virulence for these regulators (Chastanet et al., 2001; Hava \& Camilli, 2002; Hemsley et al., 2003; Lau et al., 2001; Polissi et al., 1998). One of them is encoded by $p s a R$, a transcriptional regulator responsive to $\mathrm{Mn}^{2+}$ and negatively affecting the expression of the psa operon, $p c p A, \operatorname{rlr} A$ and $p r t A$ (Johnston et al., 2006; Kloosterman et al., 2008). Recently, the PsaR-binding sequence was identified in the promoter region of $p s a B C A$, prtA and $p c p A$, and a genomewide screen for this binding sequence did not show any other putative targets (Kloosterman et al., 2008). Moreover, it has been reported that regulation by PsaR is opposite in reaction to two cations, namely repression in high $\mathrm{Mn}^{2+}$ and derepression in high $\mathrm{Zn}^{2+}$ (Kloosterman et al., 2008).

The $p c p A$ gene encodes a choline-binding protein (Sanchez-Beato et al., 1998) and rlrA encodes a transcriptional regulator, which controls the expression of the $r \operatorname{lr} A$ pathogenicity islet (Hava et al., 2003a). The psaBCA operon encodes the Psa permease that transports the cations $\mathrm{Mn}^{2+}$ and $\mathrm{Zn}^{2+}$ into the cell (Dintilhac et al., 1997; Johnston et al., 2004, 2006; Lawrence et al., 1998; McAllister et al., 2004). The permease has been implicated in virulence and protection against pneumococcal infection (Briles et al., 2000; Talkington et al., 1996). Moreover, PsaA has been shown to bind to E-cadherin, a surface molecule of the host cells (Anderton et al., 2007; Romero-Steiner et al., 2003, 2006). PsaR homologues are found in other streptococci, where they regulate homologues of $p s a B C A$ and contribute to virulence (Hanks et al., 2006; Jakubovics et al., 2000; Paik et al., 2003). The role of PsaR in pneumococcal virulence has been investigated in the strain EF3030 (serotype 19F) genetic background (Johnston et al., 2006). During 7 days of colonization no difference between the wild-type and its isogenic psaR mutant was observed, while after 7 days of lung infection, the psaR mutant was significantly attenuated compared to the wild-type (Johnston et al., 2006).
In this study, we examined the effect of the deletion of $p s a R$ on global gene and protein expression in two other pneumococcal strains, D39 (serotype 2) and TIGR4 (serotype 4), using transcriptomics and proteomics. Furthermore, we investigated the contribution of PsaR to pneumococcal virulence in three murine infection models representing the major phases in the life cycle of $S$. pneumoniae: colonization, pneumonia and bacteraemia.

\section{METHODS}

Bacterial strains and media. The pneumococcal strains used in this study are listed in Table 1; they were grown at $37{ }^{\circ} \mathrm{C}$ in Todd-Hewitt yeast broth (THY), in chemically defined medium (CDM, recipe available on request), or on Colombia base agar (Oxoid) supplemented with $5 \%$ sheep blood (Biotrading). Pneumococcal strains were maintained in $10 \%(\mathrm{v} / \mathrm{v})$ glycerol, $10 \%$ skim milk at $-80{ }^{\circ} \mathrm{C}$. Escherichia coli DH5 $\alpha$ (Stratagene) was grown in Luria broth at $37{ }^{\circ} \mathrm{C}$ with shaking or on Luria broth agar. Media were supplemented with antibiotics (50 mg ampicillin $1^{-1}$ and/or $20 \mathrm{mg}$ trimethoprim $\mathrm{l}^{-1}$ ) when appropriate.

Construction of psaR mutants. The gene encoding psaR was deleted from strain TIGR4 (sp1638) and D39 (spd1450) by allelic replacement with the $d f r 13$ cassette conferring trimethoprim resistance (Adrian et al., 2000). To this end, psaR with $1000 \mathrm{bp}$ of upstream and downstream flanking sequences was amplified from chromosomal TIGR4 DNA using primer pair psaRSacFw and psaRKnpRv (all primers are listed in Table 2). This amplicon was cloned into pBlueScript KS + (Stratagene) using the SacII and KpnI restriction sites. The coding sequence of $p s a R$ was deleted from the plasmid by performing an inverse PCR with primer pair psaRNotFw and psaRSalRv, amplifying the psaR-flanking sequences and pBlueScript KS + and introducing NotI and SalI restriction sites for further cloning. This amplicon was ligated to the $d f r 13$ cassette, which was amplified from pKOT (Hendriksen et al., 2008a) with the primers TmpSalFw and TmpNotRv, to create the knockout construct pKOpsaR-T4, and transformed into E. coli DH5 $\alpha$. A 2620 bp linear DNA fragment containing psaR-flanking DNA and $d f r 13$ was amplified from pKOpsaR-T4 using primer pair psaRSacFw and psaRKnpRv. This PCR product was used to delete $p s a R$ from the genome of S. pneumoniae TIGR4 by CSP-2-induced (100 $\mathrm{ng} \mathrm{ml}^{-1}$ ) transformation. Transformants were selected on the basis of trimethoprim resistance and were checked by sequencing for recombination at the desired location on the chromosome, i.e. replacement of $p s a R$ by $d f r 3$ (which will be transcribed in the opposite direction to $p s a R$ ). Wild-type TIGR4 was subsequently transformed with chromosomal DNA isolated from these $\Delta p s a R$ transformants to rule out the possibility of any additional mutations on the chromosome. The identical procedure was performed for the construction of D39 $\Delta p s a R$, with the exception that the $3^{\prime}$ chromosomal region from TIGR4 of pKOpsaR-T4 was replaced by the D39-specific 3' psaR region. This region differs between the two

Table 1. Pneumococcal strains used in this study

\begin{tabular}{|lccl|}
\hline Strain & Gene identifier & Antibiotic resistance & Reference \\
\hline D39 wild-type & - & - & NCTC 7466; serotype 2 \\
D39 $\Delta$ psaR & spd1450 & Trimethoprim & This study \\
TIGR4 wild-type & - & - & ATCC BAA-334; serotype 4 \\
TIGR4 $\Delta$ psaR & sp1638 & Trimethoprim & This study \\
\hline
\end{tabular}


Table 2. Oligonucleotide primers used in this study

\begin{tabular}{|llcl|}
\hline Primer name & \multicolumn{1}{c|}{ Sequence $\left(\mathbf{5}^{\prime}-\mathbf{3}^{\prime}\right)^{*}$} & Restriction site & Strain \\
\hline psaRSacFw & GCGCCCGCGGGGAATTTGCATCCTCTTCTCC & SacII & D39/TIGR4 \\
psaRKnpRv & GCGCGGTACCATATTGCCCATCAGCTTTCC & KpnI & TIGR4 \\
psaRNotFw & GCGCGCGGCCGCTCCTCAGTAACGACGAGGATTT & NotI & D39/TIGR4 \\
psaRSalRv & GCGCGTCGACGCAGGTCTATGCCAATTTCA & SalI & D39/TIGR4 \\
TmpSalFw & CGCGGTGGTCGACGGATTTTTGTGAGCTTGGACT & SalI & D39/TIGR4 \\
TmpNotRv & GGGGGGCCGCGGCCGCTTACGACGCGCATAGACG & NotI & D39/TIGR4 \\
psaRKpnRv-D39 & GAAAATGGTACCAGAGAGCAAGAGCCACTC & KpnI & D39 \\
SeqTmpFw & ATAAATGCGGACCGATTCC & - & D39/TIGR4 \\
SeqTmpRv & GCCTTCTTCCCAGTGCTTAAC & - & D39/TIGR4 \\
\hline
\end{tabular}

${ }^{\star}$ Restriction sites on oligonucleotide primers are underlined.

strains: it contains an ISS element in TIGR4, and a small ORF encoding a unique hypothetical protein in D39. Transformation of D39 was induced with CSP-1 (100 $\left.\mathrm{ng} \mathrm{m}^{-1}\right)$.

\section{Transcriptional profiling of D39 and TIGR4 psaR mutants.} Microarray analysis was performed essentially as described by Hendriksen et al. (2007, 2008a). In short, $500 \mathrm{ml} \mathrm{CDM} \mathrm{was}$ inoculated with 10-20 colonies from agar plates, and these cultures were grown statically at $37{ }^{\circ} \mathrm{C}$. Samples for RNA isolation were taken when the cultures reached an optical density $\left(\mathrm{OD}_{600}\right)$ of 0.2 (midexponential growth). RNA was isolated and purified using the High Pure RNA isolation kit (Roche Diagnostics) as described by Hendriksen et al. (2007, 2008a). Contaminating genomic DNA was removed by treatment with RNase-free DNase I (Roche Diagnostics). RNA was isolated from three replicate cultures. Synthesis, subsequent labelling of cDNA, and microarray hybridization were performed as previously described (Hendriksen et al., 2007; Kloosterman et al., 2006). In all cases, dye-swapping was performed with one of the three biological replicates. Microarrays used in this study were constructed as previously described (Hendriksen et al., 2007; Kloosterman et al., 2006) and contain amplicons representing 2087 ORFs of $S$. pneumoniae TIGR4 and 184 ORFs unique for S. pneumoniae R6, all spotted in duplicate.

The microarray data have been deposited in the NCBI Gene Expression Omnibus (GEO) database (http://www.ncbi.nlm.nih.gov/ geo/) under GEO Series accession number GSE13505.

DNA microarray data analysis. Dual-channel array images were acquired with a GeneTac LS IV confocal laser scanner (Genomics Solutions) and analysed with ArrayPro 4.5 software (Media Cybernetics). Spots were screened visually to identify those of low quality. Slide data were processed using MicroPreP as previously described (Garcia de la Nava et al., 2003; Hendriksen et al., 2007; van Hijum et al., 2003). Prior to analysis, automatically and manually flagged spots and spots with very low background-subtracted signal intensity [5\% of the weakest spots (sum of Cy 3 and Cy 5 net signals)] were filtered out of all datasets. Spots with a signal in one channel and no signal in the other were subjected to an empty-value assignment of $1 \%$, after which net signal intensities were calculated using a gridbased background subtraction. A grid-based Lowess transformation was performed for slide normalization, negative and empty values were removed, and outliers were removed by the deviation test. Further analysis was performed using a Cyber-T Student's $t$-test for paired data (Long et al., 2001). For identification of differentially expressed genes, only genes with a minimum of six reliable measurements, a Bayesian $P$-value $<0.001$, a false discovery rate $(F D R)<0.05$, and a standard deviation less than the ratio were included. Since these criteria are purely a statistical measure of differential gene expression and reproducibility across replicates, an additional fold-change cut-off of 2 was applied.

Stable isotope labelling in cell culture (SILAC). For SILAC experiments, D39 wild-type and psaR-mutant strains were inoculated in THY and grown to mid-exponential phase. These cultures were used to inoculate $\mathrm{CDM}$, supplemented with both lysine and arginine as the light $\left({ }^{12} \mathrm{C}_{6}\right.$ L-lysine, ${ }^{12} \mathrm{C}_{6}$ L-arginine; psaR mutant) or heavy $\left({ }^{13} \mathrm{C}_{6} \mathrm{~L}-\right.$ lysine, ${ }^{13} \mathrm{C}_{6} \mathrm{~L}$-arginine; wild-type) isotopic counterparts. When these cultures reached an $\mathrm{OD}_{600}$ of 0.2 , they were diluted to an $\mathrm{OD}_{600}$ of 0.04 in fresh pre-warmed CDM with the appropriate heavy or light lysine and arginine, and grown to an $\mathrm{OD}_{600}$ of 0.2. This was repeated until the cells had been grown in heavy or light lysine- and arginine-containing $\mathrm{CDM}$ for at least five or six generations. After the last generation, the cells were harvested by centrifugation and washed once with PBS. Equal amounts of heavy-labelled wild-type and light-labelled mutant cells were combined and used for mass spectrometry. Bacterial pellets were lysed in lysis buffer [7 M urea, $2 \mathrm{M}$ thiourea, protease inhibitor mix (Roche), $\mathrm{pH}$ 8.0]. Lysates were subjected to reduction and alkylation using dithiothreitol and iodoacetamide before LysC and trypsin digestion. Peptide mixtures were purified and desalted using $\mathrm{C}_{18}$-stage tips. Peptide separation and sequence determination was performed with a nano-high-performance liquid chromatography system (Agilent 1100 series) connected to a 7-T linear quadrupole ion trap ion cyclotron resonance Fourier transform mass spectrometer (Thermo Electron). Peptides were separated on a $15 \mathrm{~cm} 100 \mu \mathrm{m}$ inner diameter PicoTip emitter for online electrospray (New Objective) packed with $3 \mu \mathrm{m} \mathrm{C18}$ beads (Reprosil, Dr Maisch $\mathrm{GmbH}$ ) with a 60 min linear gradient from 2.4 to $40 \%$ acetonitrile in $0.5 \%$ acetic acid at a flow rate of $300 \mathrm{nl} \mathrm{min}{ }^{-1}$. The four most abundant ions were sequentially isolated and fragmented in the linear ion trap by applying collisionally induced dissociation. Proteins were identified using the MASCOT search engine (Matrix Science) against the corresponding S. pneumoniae database. MSQuant was used for the quantification and determination of peptide ratios between wild-type and $p s a R$ mutant. A protein was considered differentially expressed when the results of three technical replicates showed at least a 1.5 -fold change in protein abundance and a $P$-value $<0.05$ (one-sample $t$-test).

Infection models. Nine-week-old female outbred CD-1 mice (Harlan) were used for all infection models. Prior to the infection experiments, D39 and TIGR4 (wild-type and psaR mutants) were passaged in mice as described previously (Kerr et al., 2004). Cultures of S. pneumoniae D39 and TIGR4 (wild-type and $\Delta p s a R$ ) were grown in THY broth to an $\mathrm{OD}_{600}$ of 0.3 , and stored in aliquots in $10 \%$ glycerol at $-80{ }^{\circ} \mathrm{C}$. Prior to infection, these aliquots were spun down and bacteria were resuspended in sterile PBS to $10^{6}$ c.f.u. in volumes 
Table 3. Differentially expressed genes/proteins in $\Delta p s a R$ of D39 and/or TIGR4

Ratios are given as $\log _{2}$-transformed expression of $\Delta p s a R /$ wild-type.

\begin{tabular}{|c|c|c|c|c|c|c|c|}
\hline \multicolumn{2}{|c|}{ Identifier } & \multirow[t]{2}{*}{ Gene } & \multirow[t]{2}{*}{ Annotation (http://www.kegg.com) } & \multicolumn{2}{|c|}{ Microarray } & \multicolumn{2}{|c|}{ SILAC } \\
\hline TIGR4 & D39 & & & TIGR4 & D39 & TIGR4 & D39 \\
\hline \multicolumn{8}{|c|}{ Upregulated } \\
\hline sp0232 & spd0215 & $\operatorname{infA}$ & Translation initiation factor IF-1 & & & & 2.4 \\
\hline sp0303 & spd0277 & bglA & 6-Phospho- $\beta$-glucosidase & & 1.1 & & \\
\hline sp0306 & spd0280 & & Transcriptional regulator, putative & & 1.2 & & \\
\hline sp0308 & spd0281 & & PTS system, cellobiose-specific IIA component & & 1.5 & & \\
\hline sp0310 & spd0283 & & PTS system, cellobiose-specific IIC component & & 1.2 & & \\
\hline- & spd0322 & $\operatorname{cps} 2 G$ & Glycosyltransferase, group 1 family protein & & & & 4.5 \\
\hline sp0459 & spd0420 & $p f l B$ & Formate acetyltransferase & & & 0.8 & \\
\hline sp0461 & - & $r l r A$ & Transcriptional regulator & 2.7 & & & \\
\hline spo462 & - & $\operatorname{rrgA}$ & Cell wall surface anchor family protein & 3.1 & & & \\
\hline sp0463 & - & $\operatorname{rrgB}$ & Cell wall surface anchor family protein & 3.7 & & 2.0 & \\
\hline sp0464 & - & $r r g C$ & Cell wall surface anchor family protein & 3.7 & & & \\
\hline sp0467 & - & & Sortase SrtC, putative & 2.5 & & & \\
\hline spo468 & - & & Sortase SrtD, putative & 2.6 & & & \\
\hline sp0641 & spd0558 & prtA & Serine protease, subtilase family PrtA & 3.6 & 3.0 & & 3.9 \\
\hline spo869 & spd0764 & sufS & Cysteine desulfurases, SufS subfamily protein & & & & 5.0 \\
\hline sp1244 & spd1101 & $f t s Y$ & Signal recognition particle-docking protein FtsY & & & & 7.6 \\
\hline sp1517 & spd1345 & greA & Transcription elongation factor GreA & & & & 2.9 \\
\hline sp1531 & spd1360 & & Hypothetical protein & & & & 2.7 \\
\hline sp1636 & spd1448 & & Rrf2 family protein & 1.3 & & & \\
\hline sp1637 & spd1449 & & Hypothetical protein & 1.4 & 1.2 & & \\
\hline sp1647 & spd1460 & рерO & Endopeptidase O & & & 0.6 & \\
\hline sp1648 & spd1461 & psaB & Manganese $\mathrm{ABC}$ transporter, ATP-binding protein & 3.6 & 3.3 & & \\
\hline sp1649 & spd1462 & psaC & Manganese ABC-transporter permease & 2.8 & 2.4 & & \\
\hline sp1650 & spd1463 & psaA & $\mathrm{ABC}$ transporter, substrate-binding lipoprotein & & & 5.4 & 5.1 \\
\hline sp1805 & spd1591 & & Hypothetical protein & & & 0.6 & \\
\hline sp1856 & spd1637 & & Transcriptional regulator, MerR family & 1.2 & & & \\
\hline sp1857 & spd1638 & $c z c D$ & Cation-efflux system protein & 1.7 & & & \\
\hline sp2136 & spd1965 & $p c p A$ & Choline-binding protein PcpA & 4.0 & 4.6 & 7.4 & \\
\hline sp2210 & spd2037 & cysK & Cysteine synthase A & & & & 4.0 \\
\hline \multicolumn{8}{|c|}{ Downregulated } \\
\hline sp0112 & spd0109 & & $\begin{array}{l}\text { Polar amino acid transport system substrate-binding } \\
\text { protein }\end{array}$ & & -1.2 & & \\
\hline sp0138 & spd0141 & & Hypothetical protein & & -1.7 & & \\
\hline sp0139 & spd0142 & & Hypothetical protein & & -1.8 & & \\
\hline spo140 & spd0143 & ugd & UDP-glucose/GDP-mannose dehydrogenase & & -1.7 & & \\
\hline spo141 & spd0144 & mutR & Positive transcriptional regulator of MutA & & -1.2 & & \\
\hline sp0142 & spd0145 & & Hypothetical protein & & -1.0 & & \\
\hline spo143 & spd0146 & & Hypothetical protein & & -1.9 & & \\
\hline sp0144 & spd0147 & & Hypothetical protein & & -1.5 & & \\
\hline sp0145 & spd0148 & & Hypothetical protein & & -2.0 & & \\
\hline sp0146 & spd0149 & & Hypothetical protein & & -1.7 & & \\
\hline sp0148 & spd0150 & & $\mathrm{ABC}$ transporter, substrate-binding protein & & & -2.1 & \\
\hline sp0524 & spd0466 & & BlpT protein, fusion & & -1.5 & & \\
\hline sp0525 & spd0467 & $b l p S$ & BlpS protein & & -1.4 & & \\
\hline sp0526 & spd0468 & $b l p R$ & Response regulator BlpR (TCS13) & & -1.2 & & \\
\hline sp0527 & spd0469 & $b l p H$ & Sensor histidine kinase BlpH, putative (TCS13) & & -1.1 & & \\
\hline sp0529 & spd0471 & $b l p B$ & BlpC ABC transporter & & -1.5 & & \\
\hline sp0530 & spd0472 & $b l p A$ & BlpA, pseudogene & & -1.5 & & \\
\hline sp0533 & spd0046 & $b l p K$ & Bacteriocin BlpU (highly similar to sp0533) & & -1.5 & & \\
\hline sp0541 & spd0046 & $b l p O$ & Bacteriocin BlpO (5' highly similar to sp0541) & & -1.5 & & \\
\hline spo545 & spd0473 & $b l p Y$ & Immunity protein BlpY & & -2.4 & & \\
\hline sp0546 & spd0474 & $b l p Z$ & BlpZ protein, fusion & & -1.2 & & \\
\hline
\end{tabular}


Table 3. cont.

\begin{tabular}{|c|c|c|c|c|c|c|c|}
\hline \multicolumn{2}{|c|}{ Identifier } & \multirow[t]{2}{*}{ Gene } & \multirow[t]{2}{*}{ Annotation (http://www.kegg.com) } & \multicolumn{2}{|c|}{ Microarray } & \multicolumn{2}{|c|}{ SILAC } \\
\hline TIGR4 & D39 & & & TIGR4 & D39 & TIGR4 & D39 \\
\hline sp0664 & spd0577 & $z m p B$ & Zinc metalloprotease $\mathrm{ZmpB}$, putative & & & -0.8 & \\
\hline sp0798 & spd0701 & $\operatorname{ciaR}$ & DNA-binding response regulator CiaR & & -1.1 & & \\
\hline sp0925 & spd0817 & & Hypothetical protein & & -1.4 & & \\
\hline sp1249 & spd1107 & & GMP reductase & -1.0 & & & \\
\hline sp1458 & spd1287 & $\operatorname{trx} B$ & Thioredoxin reductase & & & -1.0 & \\
\hline sp1543 & spd1372 & & Glyoxalase family protein & & -1.5 & & \\
\hline sp1638 & spd1450 & $p s a R$ & Iron-dependent transcriptional regulator, PsaR & -2.7 & -2.0 & & \\
\hline sp1802 & spd1588 & & Hypothetical protein & & -1.2 & & \\
\hline sp1804 & spd1590 & & General stress protein 24 , putative & & -1.3 & & \\
\hline
\end{tabular}

depending on the infection model used. Upon intranasal infection, mice were anaesthetized with $2.5 \%(\mathrm{v} / \mathrm{v})$ isoflurane $/ \mathrm{O}_{2}$. At predetermined time points after infection, groups of mice were sacrificed by cervical dislocation and samples of various sites were taken to determine the bacterial load. During infection, signs of disease were closely monitored. If animals reached a moribund state, they were sacrificed by cervical dislocation and excluded from the experiment prematurely. All animal experiments were performed with approval from the Animal Experimentation Committee (DEC) of Erasmus Medical Centre, Rotterdam, The Netherlands.

Colonization model of infection. In the colonization model, $10 \mu \mathrm{l}$ PBS containing $10^{6}$ c.f.u. bacteria was administered to the nostrils of groups of five mice as described previously (Hendriksen et al., 2008b; Kadioglu et al., 2000). Due to this small volume, only the nose of the mice becomes infected. Bacteria were recovered from the nasopharynx by flushing the nose with $2 \mathrm{ml}$ sterile PBS (Kerr et al., 2004), and lungs were removed from the body and homogenized in $2 \mathrm{ml}$ sterile PBS using a hand-held homogenizer (Polytron PT 1200, Kinematica $A G)$. Viable bacteria from the nasal lavage fluid and homogenized lung samples were counted by plating serial 10-fold dilutions on Colombia blood agar plates. Time points for sampling were $30 \mathrm{~min}$, $24 \mathrm{~h}, 48 \mathrm{~h}, 96 \mathrm{~h}$ and $192 \mathrm{~h}$ post-infection. The $30 \mathrm{~min}$ time point is considered to be the start of the infection, and is therefore referred to as $t=0$. Bacteriology results are expressed as geometric mean \pm SEM. Comparison of bacterial loads in the time-course experiment was performed using a Student's $t$-test with $P<0.05$ considered statistically significant.

Pneumonia model of infection. In the pneumonia model, five mice per group were infected with $50 \mu \mathrm{l}$ PBS containing $10^{6}$ c.f.u. pneumococci as described previously (Hendriksen et al., 2008b). Bacteria were recovered from the different sites as described above, with the addition of a blood sample obtained by cardiac puncture. Time points for sampling were $0,12,24$ and $36 \mathrm{~h}$ post-infection. Viable bacteria isolated from the nasal lavage fluid, homogenized lungs and blood were quantified as described above. Bacteriology results are expressed as geometric mean \pm SEM. Comparison of bacterial loads in the time-course experiment was performed using a Student's $t$-test with $P<0.05$ considered statistically significant.

Bacteraemia model of infection. In the bacteraemia model, groups of ten mice were infected in a tail vein with $10^{6}$ c.f.u. resuspended in
$100 \mu \mathrm{l}$ sterile PBS as described previously (Hendriksen et al., 2008b). Bacteria were recovered from the blood by a lateral tail vein puncture from the same mouse at three predetermined time points after infection $(0,12,24 \mathrm{~h})$ and by a cardiac puncture at the last time point, $36 \mathrm{~h}$. In addition, mouse survival times were scored, after which analysis of survival times was performed using the log-rank test with $P<0.05$ considered statistically significant.

\section{RESULTS}

\section{Transcriptional analyses of $\Delta p s a R$ in D39 and TIGR4}

By means of microarrays analysis, we assessed which genes were affected in expression due to the mutation of $p s a R$ in two genetic backgrounds, i.e. D39 and TIGR4. To this end, transcriptional profiles of wild-type strains were compared to their isogenic $\Delta p s a R$ strains. These bacteria were grown in chemically defined medium $(\mathrm{CDM})$ and harvested at mid-exponential growth phase. In all experiments, the $p s a R$ mutant strains grew like the wild-type. The concentration of $\mathrm{Mn}^{2+}$ in CDM is $180 \mu \mathrm{M}$, which is sufficient for PsaR regulation (see Kerr et al., 2004, where $50 \mu \mathrm{M}$ was used). For comprehensibility, loci of D39 are referred to by the TIGR4 gene identifiers (in Table 3 both annotations are given). Comparison of transcriptional profiles of D39 and TIGR4 wild-type with their $\Delta p s a R$ counterparts revealed 19 differentially expressed genes in TIGR $4 \Delta p s a R$, and 37 in D $39 \Delta p s a R$. Of these, five genes were upregulated in both TIGR4 and D39 psaR mutants, while only psaR itself was downregulated in both strains (Fig. 1).

The genes that were differentially expressed in both serotypes were all upregulated in the $p s a R$ mutant, confirming the general role of PsaR as a transcriptional repressor (Johnston et al., 2006; Kloosterman et al., 2008). Among those were the previously described targets, the $p s a$ operon (sp1648-sp1650), pcpA (sp2136) and prtA (sp0614). 


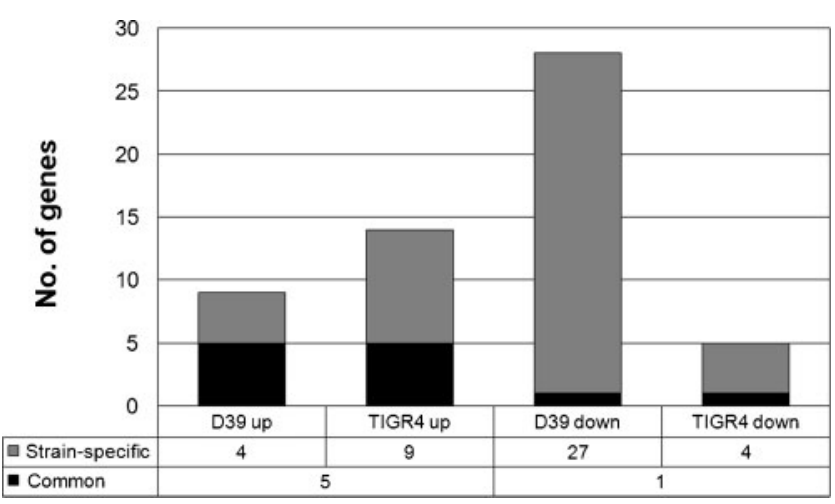

Fig. 1. Number of genes differentially expressed in $\mathrm{D} 39 \Delta p s a R$ and TIGR4 $\Delta p s a R$.

In addition, sp1637, encoding a hypothetical protein of unknown function, was found to be upregulated in the psaR mutants of both strains (Table 3).

Four genes were found to be repressed by PsaR in D39 (i.e. upregulated in D39 $\Delta p s a R$ ) only: sp0303, encoding 6phospho- $\beta$-glucosidase; sp0306, encoding a putative transcriptional regulator; and two genes of a putative operon encoding a cellulose-specific phosphotransferase system (PTS) (sp0308 and sp0310).

Twenty-seven genes were downregulated in D39 $p$ psaR. This set of genes contained sp0112, predicted to encode an amino acid substrate-binding protein; the transcriptional regulator $m u t R$ (sp0141); a putative bacteriocin system (sp0142-sp0146); the blp two-component system (TCS13; sp0526-sp0527); the gene encoding response regulator CiaR (sp0798); and glyA (sp1024) encoding serine hydroxymethyltransferase. The full set of differentially expressed genes in D39 is listed in Table 3.

In addition to the common gene targets, nine genes were specifically upregulated in the psaR mutant in TIGR4 (Fig. 1). These included the $r l r A$ pathogenicity islet (sp0461-sp0468) as reported previously (Johnston et al., 2006); sp1636 (encoding an Rf2 family protein); and two adjacent genes, encoding a MerR family transcriptional regulator (sp1856) and $c z c D$ (sp1857), a $\mathrm{Zn}^{2+}$-efflux pump (Table 3).

Four genes were downregulated: guaC, encoding GMP reductase (sp1249); ply, encoding pneumolysin (sp1923); and two genes encoding the $\mathrm{B}$ and $\mathrm{C}$ components of a putative cellobiose-specific PTS (sp2022 and sp2023). The complete set of differentially expressed genes in TIGR4 $\Delta p s a R$ is given in Table 3.

\section{Proteome analysis of $\triangle p s a R$ in D39 and TIGR4}

To examine if the observed PsaR-mediated differences in gene expression corresponded to changes in protein expression, we performed SILAC for both wild-type strains and their psaR mutants. To this end, wild-type and isogenic
$\Delta p s a R$ counterparts were cultured in the presence of stable isotope-labelled (heavy, ${ }^{13} \mathrm{C}_{6}$ ) or normal (light, ${ }^{12} \mathrm{C}_{6}$ ) Llysine and L-arginine, respectively. Equal amounts of bacteria were mixed and analysed by mass spectrometry, after which changes in protein expression between wildtype and isogenic $\triangle p s a R$ strains were derived from the corresponding heavy to light peptide ratios.

In D39 $\Delta p s a R$, nine proteins were found to be more abundant than in the wild-type (Table 3). Most pronounced were FtsY, PsaA, SufS, Cps2G, CysK and PrtA, all of which displayed expression levels at least eight times higher in the psaR mutant than in the wild-type. No proteins were identified that were less abundant in D39 $\Delta p s a R$ (Table 3).

In TIGR4 $\Delta p s a R$, six proteins were more abundant than in the wild-type. Most pronounced were PcpA, PsaA and $\mathrm{RrgB}$, all with at least a fourfold increase in expression in the psaR mutant (Table 3). Three proteins were less abundant in TIGR $4 \Delta p s a R$, i.e. an $\mathrm{ABC}$ transporter of a putative bacteriocin system (sp0148), TrxB and ZmpB (Table 3).

\section{PsaR does not contribute to pneumococcal colonization}

To assess the contribution of PsaR regulation to pneumococcal virulence, we examined the phenotypes of the two wild-type strains and their $p s a R$ mutants in three murine models of infection. In the colonization model, both wildtype D39 and TIGR4 were capable of extended colonization of the murine nasopharynx for a period of $192 \mathrm{~h}$. The level of colonization was fairly consistent during this period, varying between $10^{4}$ and $10^{6}$ c.f.u. $\mathrm{ml}^{-1}$ (Fig. 2).

We did not observe a clear effect of the deletion of psaR on the colonization ability of strains: comparable bacterial loads of wild-type and mutant strains were found during $192 \mathrm{~h}$ of colonization, with bacterial loads between $2.5 \times 10^{4}$ c.f.u. $\mathrm{ml}^{-1}$ and $3.2 \times 10^{5}$ c.f.u. $\mathrm{ml}^{-1}$ for D39 wild-type and its isogenic psaR mutant, and between $1.3 \times 10^{5}$ c.f.u. $\mathrm{ml}^{-1}$ and $5.0 \times 10^{5}$ c.f.u. $\mathrm{ml}^{-1}$ for TIGR4 wild-type and its $\Delta p s a R$ derivative (Fig. $2 \mathrm{a}$ and b). The only exception was a small but significantly higher load $(P=0.0049)$ of D39 $\Delta p s a R$ at 24 h (Fig. 2b).

\section{PsaR is not required for pneumococcal pneumonia}

In the pneumonia model, we observed a similar trend in bacterial loads of the nasopharynx as was observed in the colonization model, although the actual numbers of bacteria were lower. At $12 \mathrm{~h}$ of infection there was a small but significantly higher nasopharyngeal load in mice infected with $\mathrm{D} 39 \Delta p s a R$ as compared to wild-type (data not shown). For TIGR4 wild-type and $\Delta p s a R$, small but significant differences in bacterial load were observed throughout the entire experiment (data not shown). 


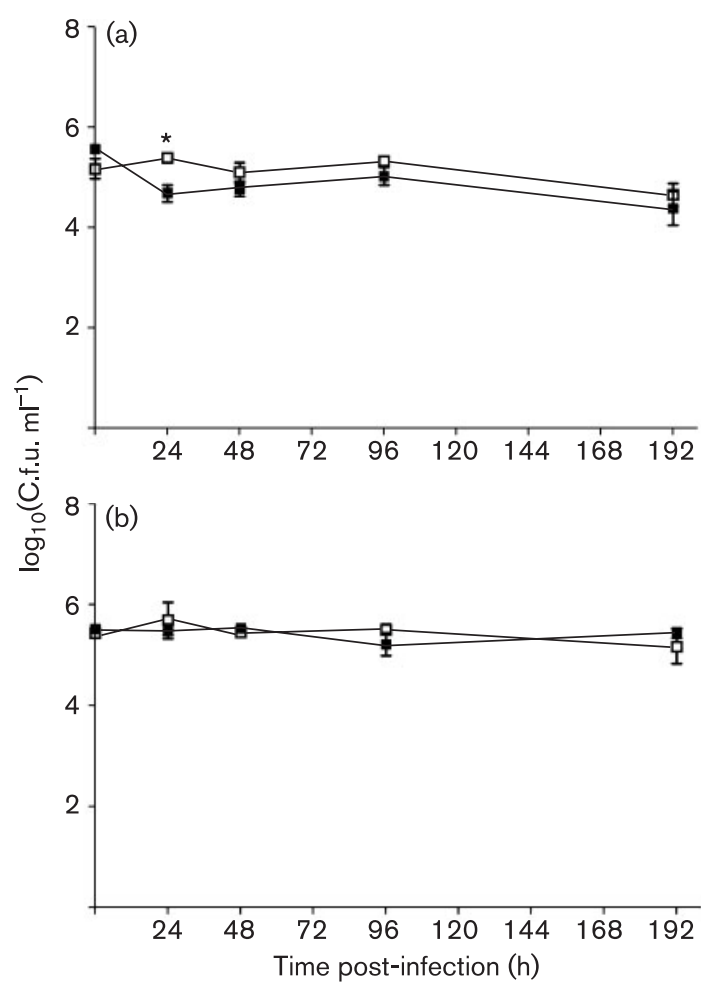

Fig. 2. Colonization model. Bacterial loads in the nasal lavage fluid of mice infected with (a) D39 wild-type ( $\boldsymbol{\square}$ ) and D39 $\Delta$ psaR ( $\square$ ), (b) TIGR4 wild-type ( $\square$ ) and TIGR4 $\Delta p s a R(\square) .{ }^{*}, P<0.05$.

Furthermore, bacterial loads in the nasopharynx were comparable to those of the first $36 \mathrm{~h}$ in the colonization model (data not shown).

We did not observe any clear difference in the bacterial survival in the lungs between wild-type and $p s a R$ mutants (Fig. 3a). However, immediately after infection $(0 \mathrm{~h})$ and $12 \mathrm{~h}$ post-infection, the lung homogenates of D39 $\mathrm{p} p s a R$ infected mice had significantly higher bacterial loads than those of wild-type infected mice: $1.2 \times 10^{5}$ vs $6.3 \times$ $10^{4}$ c.f.u. $\mathrm{ml}^{-1}$ at $0 \mathrm{~h}$, and $1.5 \times 10^{5}$ vs $5.8 \times 10^{4}$ c.f.u. $\mathrm{ml}^{-1}$ at $12 \mathrm{~h}$. This suggests that there might be an initial positive effect of the $p s a R$ mutation, as the inoculum of the wild-type contained (more than) twice as many bacteria as that of the $p s a R$ mutant $\left(1.2 \times 10^{6}\right.$ and $4.5 \times 10^{5}$ c.f.u. $\mathrm{ml}^{-1}$, respectively).

In contrast, mice infected with the TIGR4 psaR mutant had lower bacterial loads in the lungs compared to mice infected with TIGR4 wild-type, although these differences were only statistically significant at $24 \mathrm{~h}$ post-infection $(P=0.0401)$ (Fig. 3b).

The number of bacteria that were able to reach the systemic circulation was not significantly different between wildtype and $\Delta p s a R$ in either D39 or TIGR4 (data not shown), indicating that PsaR regulation is not required for the transition from the lungs to the systemic circulation.

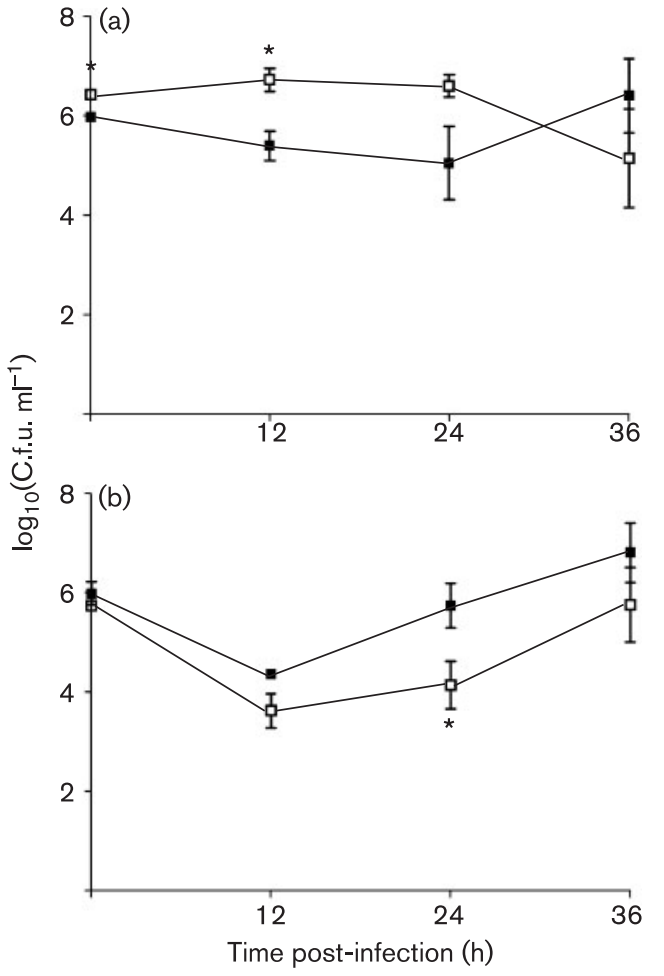

Fig. 3. Pneumonia model. Bacterial loads in lungs and blood of mice infected with (a) D39 wild-type ( $\square$ ) and D39 $\Delta p s a R(\square)$, (b)

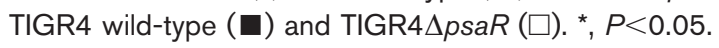

\section{PsaR contributes to survival of pneumococci during bacteraemia}

The most prominent phenotype of the $p s a R$ mutants was observed in the bacteraemia model of infection. D39 wildtype-infected mice had significantly more bacteria in the blood than the D39 $\Delta p s a R$-infected mice during the first $24 \mathrm{~h}$ of infection (Fig. $4 \mathrm{a}$ ), while variation in the inocula was marginal $\left(1.6 \times 10^{6}\right.$ for wild-type and $1.4 \times 10^{6}$ for psaR mutant). In contrast, no differences were seen between mice infected with TIGR4 wild-type and TIGR $4 \Delta p s a R$ during the first $24 \mathrm{~h}$ of infection. At $36 \mathrm{~h}$ post-infection, however, TIGR4 wild-type-infected mice had twice as many bacteria in the bloodstream $(P=0.0396)$ (Fig. 4b).

In addition to the differences in bacterial load, we also observed a difference in murine survival after infection with D39 or TIGR4 in our bacteraemia model: all TIGR4infected mice (wild-type and $\Delta p s a R$ ) survived, in contrast to D39 wild-type infected mice, which died within $30 \mathrm{~h}$. Of the D39 $\Delta p s a R$-infected mice, three survived the experiment. Consequently, the median survival time of D39 $\Delta p s a R$-infected mice was significantly longer than that of mice infected with its wild-type parental strain (the median survival for $\Delta p s a R$ was $24 \mathrm{~h}$ and for wild-type $30 \mathrm{~h}$, with $P=0.0405$ and hazard ratio $=1.866$ ). 


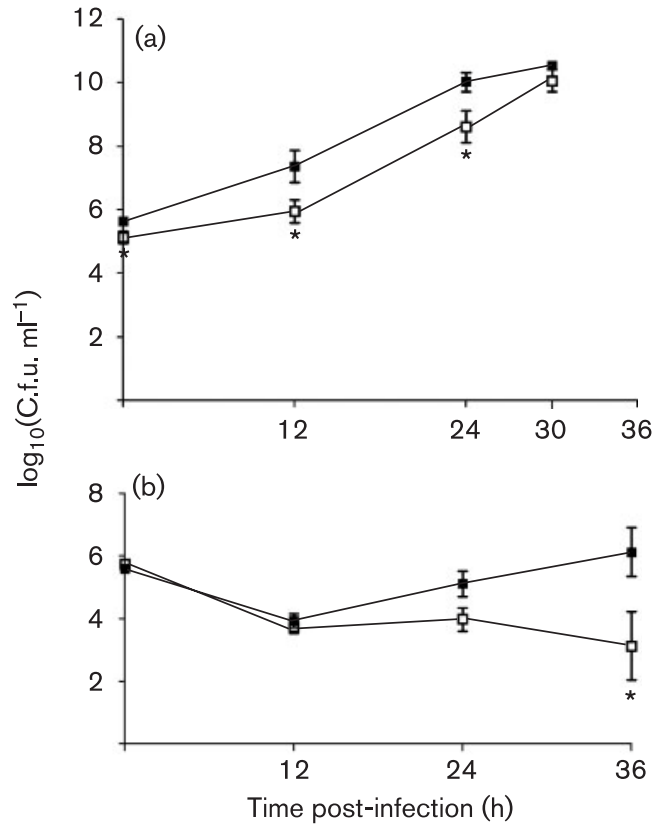

Fig. 4. Bacteraemia model. Bacterial loads in blood of mice infected with (a) D39 wild-type ( $\square$ ) and D39 $\Delta p s a R(\square)$, (b) TIGR4 wild-type ( $\square$ ) and TIGR4 $\Delta$ psaR $(\square)$. ${ }^{*}, P<0.05$.

\section{DISCUSSION}

During colonization and infection of the human host, $S$. pneumoniae encounters fluctuating amounts of free $\mathrm{Mn}^{2+}$ in the different niches where the bacterium resides. Since $\mathrm{Mn}^{2+}$ is required for several cellular processes, proper regulation of manganese homeostasis is vital for pneumococcal physiology and virulence. The transcriptional repressor PsaR has been described to play an important role in this process, at least in the genetic background of strain EF3030 (serotype 19F) (Johnston et al., 2006). However, we and others have previously shown that, even though transcriptional regulators themselves appear to be conserved between S. pneumoniae strains, they often have a strain-specific impact on global transcription and virulence (Hendriksen et al., 2007; Blue \& Mitchell, 2003; McCluskey et al., 2004). To examine whether a similar strain specificity holds true for PsaR, we identified its targets and its contribution to experimental virulence in two additional strains, TIGR4 and D39.

We used a combination of transcriptional and proteome analyses for the identification of PsaR targets. Observed discrepancies between these two approaches could be the result of several factors, such as low levels of gene expression, protein instability, or post-translational regulation. The previously reported PsaR targets, the Psa operon, $p c p A$ and prtA (Johnston et al., 2006; Kloosterman et al., 2008), were confirmed in TIGR4 and D39 by both transcriptomics and proteomics. In addition, we found sp1637, encoding a hypothetical protein, to be upregulated in both $p s a R$ mutants. Since the latter gene is located directly upstream of $p s a R$ ( $s p 1638$ ), we cannot entirely exclude the possibility that the derepression of sp1637 is caused by transcriptional read-through of the trimethoprim-resistance cassette used to create the $p s a R$ knockout. We have previously shown that the common PsaR target $p c p A$ is required for adherence to the human Detroit epithelial cell line and that the expression of this gene is also directly positively regulated by the nutritional regulator CodY (Hendriksen et al., 2008a). The inability of the $\operatorname{cod} Y$ mutant to colonize the murine nasopharynx underscored, albeit indirectly, the involvement of PcpA in adherence and colonization. However, a recent study showed that PcpA is not involved in colonization but has a role in invasive disease (Glover et al., 2008). These contradictory results are possibly due to different experimental set-ups; however, the exact role of PcpA during pneumococcal pathogenesis remains unclear.

In addition to the shared PsaR targets (i.e. the overlapping genes between D39 and TIGR4), several genes were differentially expressed in D39 or TIGR4 only. This strain-specific differential expression might be due to direct regulation by PsaR, but is more likely to be either an indirect effect caused by an imbalance in $\mathrm{Mn}^{2+} / \mathrm{Zn}^{2+}$ homeostasis due to the lack of PsaR or, possibly, downstream signalling of other regulators. Moreover, it seems that this effect was more severe for D39, since more genes and proteins were affected in their expression level in this strain. Downstream regulation or a disturbed balance in other cellular processes might be the cause of this differential expression, since manganese cations have been shown to be required for several bacterial cellular processes (Jakubovics \& Jenkinson, 2001). For example, manganese cations have been shown to function as cofactors for enzymes in glycolysis, amongst others 6-phospho- $\beta$ glucosidase (Varrot et al., 1999). The genes encoding 6-phospho- $\beta$-glucosidase and a PTS downstream of it (sp0303, sp0306 and sp0308), were upregulated in $\mathrm{D} 39 \Delta p s a R$, in line with indirect regulation. However, in D39 these genes are strongly downregulated in the presence of $\mathrm{Zn}^{2+}$, which is not in agreement with the opposite effect of $\mathrm{Mn}^{2+}$ and $\mathrm{Zn}^{2+}$ on PsaR regulation (Kloosterman et al., 2008).

In $\mathrm{D} 39 \Delta p s a R$, the gene ciaR, involved in competence development, was upregulated. It has been reported that $\mathrm{Mn}^{2+}$ is required for genetic transformation (Dintilhac et al., 1997), suggesting that downregulation of this gene might be an indirect effect of the psaR mutation. Several other systems were downregulated in D39 $\Delta p s a R$ alone, such as a putative bacteriocin system (sp0142-sp0146), the blp bacteriocin system, and the blp two-component system (TCS13). The genes sp0142-sp0146 have also been shown to be regulated by the nutritional regulator CodY in D39 (Hendriksen et al., 2008a). Possibly, the downregulation of the $b l p$ bacteriocin genes is due to downregulation of the blp two-component system (Dawid et al., 2007; de Saizieu et al., 2000). 
Notably in TIGR4, a MerR family regulator (sp1856) and $c z c D$ (sp1857), encoding a $\mathrm{Zn}^{2+}$-efflux system, were upregulated in the $p s a R$ mutant. These two genes have also been shown to be upregulated in the presence of $\mathrm{Zn}^{2+}$, underscoring the reported opposite effect of $\mathrm{Mn}^{2+}$ and $\mathrm{Zn}^{2+}$ on PsaR regulation (Kloosterman et al., 2008). The $c z c D$ gene has recently been shown to be regulated by SczA (sp1858) in reaction to increasing cellular zinc concentrations (Kloosterman et al., 2007), but this efflux system might also be involved in $\mathrm{Mn}^{2+}$ homeostasis. This again indicates that these regulatory systems of cation homeostasis are intertwined (Kloosterman et al., 2008).

The expression of ply (encoding the pneumococcal toxin pneumolysin) was downregulated as a result of the $p s a R$ mutation during in vitro growth in TIGR4 only. If this downregulation also occurred during our infection experiments, it did not have a large effect on experimental virulence of the TIGR4 strain, as the $\Delta p s a R$ mutant was as virulent as the wild-type in all our infection models. Only at $24 \mathrm{~h}$ post-infection during experimental pneumonia did mice infected with the $p s a R$ mutant have significantly lower bacterial loads in the lungs compared to those infected with the wild-type.

A role for PsaR in virulence has been reported in a strain EF3030 genetic background (Johnston et al., 2006). During 7 days of colonization no difference between the wild-type and isogenic $p s a R$ mutant was observed, which is in agreement with our results. Johnston et al. (2006) reported that during lung infection the $p s a R$ mutant had significantly lower bacterial loads than the wild-type after 7 days of infection. However, in our pneumonia model, we observed higher bacterial loads in D39 $\Delta p s a R$-infected mice at the beginning of the experiment (the first $12 \mathrm{~h}$ ). The TIGR4 $\triangle p s a R$-infected mice had significantly lower bacterial loads at $24 \mathrm{~h}$ only. Taking the results together, we did not observe any clear role for PsaR during lung infection in our study.

Upon intravenous infection, we observed the most pronounced effect of deletion of $p s a R$. The $p s a R$ mutant was attenuated at the early stages of blood infection. In line with this, PsaR was identified by a large signature-tagged mutagenesis study to be required for full virulence of a serotype 3 strain in a bacteraemia model of infection after $24 \mathrm{~h}$ infection (Lau et al., 2001). Importantly, for D39 no significant difference was observed in our model at $30 \mathrm{~h}$ post-infection. This indicates that in the first $24 \mathrm{~h} \mathrm{PsaR}$ regulation is required for adaptation to the blood. Furthermore, in D39, survival times of wild-type and $\Delta p s a R$-infected mice were different, although this just reached statistical significance. In contrast, mice infected with TIGR4 wild-type and $\Delta p s a R$ did not exhibit any differences in the first $24 \mathrm{~h}$ of experimental bacteraemia, while after $36 \mathrm{~h}$ they did. This indicates that in TIGR4, PsaR contributes to the later stages of bacteraemia. However, since the TIGR4 wild-type was not able to cause infection as severe as D39, this contribution is considered to be marginal.
Although we observed disturbed gene regulation in the D39 and TIGR4 mutants, in most infection models (described above) we did not observe large effects on virulence. A study performed on the pneumococcal carbohydrate regulator RegM showed that, even though deletion of a regulator does not necessarily lead to large differences in expression of known virulence genes, it can affect pneumococcal virulence (Giammarinaro \& Paton, 2002). Conversely, a second study by Trombe and co-workers showed that deleting the global regulator RegR does not dramatically affect virulence, but has a large effect on gene expression (Chapuy-Regaud et al., 2003). It seems that the pneumococcus has several compensatory virulence pathways.

Strain-specific contributions of genes encoding transcriptional regulators have been reported previously and complicate ascribing a general role for these genes in transcriptional control of their targets and their contribution to virulence (Hendriksen et al., 2007; McCluskey et al., 2004). PsaR directly controls the expression of a select group of genes individually important for virulence (psaBCA and rlrA) (Johnston et al., 2006; Kloosterman et al., 2008). The observed differences in PsaR-regulated gene expression between D39 and TIGR4 might be the indirect effect of an unbalanced $\mathrm{Mn}^{2+}$ homeostasis, which suggests that physiological differences might reflect genetic differences present in these strains. A clear example of the genetic difference is the $r l r A$ pathogenicity islet, which is strongly upregulated in TIGR4 $\Delta p s a R$. However, the genetic diversity between strains only varies as much as $10 \%$, most of which can be attributed to the capsular genes (Hakenbeck et al., 2001; Lanie et al., 2007). In conclusion, PsaR does not contribute to colonization of S. pneumoniae, but it is involved in invasive disease where it has a strainspecific impact during both pneumonia and bacteraemia.

\section{ACKNOWLEDGEMENTS}

W. T. H. is supported by the Sophia foundation for Medical Research (SSWO 356, Rotterdam, The Netherlands) and H. J. B. is supported by IOP Genomics grant IGE03002 of the Dutch Ministry of Economic Affairs. We thank Theo Hoogenboezem, Bedette van de Zande, Anne de Jong and Lambert Lambooy for technical assistance and Tomas Kloosterman for helpful discussion.

\section{REFERENCES}

Adrian, P. V., Thomson, C. J., Klugman, K. P. \& Amyes, S. G. (2000). New gene cassettes for trimethoprim resistance, $d$ fr 13, and streptomycin-spectinomycin resistance, aadA4, inserted on a class 1 integron. Antimicrob Agents Chemother 44, 355-361.

Anderton, J. M., Rajam, G., Romero-Steiner, S., Summer, S., Kowalczyk, A. P., Carlone, G. M., Sampson, J. S. \& Ades, E. W. (2007). E-cadherin is a receptor for the common protein pneumococcal surface adhesin A (PsaA) of Streptococcus pneumoniae. Microb Pathog 42, 225-236.

Archibald, F. S. \& Fridovich, I. (1981). Manganese, superoxide dismutase, and oxygen tolerance in some lactic acid bacteria. J Bacteriol 146, 928-936. 
Blue, C. E. \& Mitchell, T. J. (2003). Contribution of a response regulator to the virulence of Streptococcus pneumoniae is strain dependent. Infect Immun 71, 4405-4413.

Bogaert, D., De Groot, R. \& Hermans, P. W. (2004). Streptococcus pneumoniae colonisation: the key to pneumococcal disease. Lancet Infect Dis 4, 144-154.

Briles, D. E., Ades, E., Paton, J. C., Sampson, J. S., Carlone, G. M., Huebner, R. C., Virolainen, A., Swiatlo, E. \& Hollingshead, S. K. (2000). Intranasal immunization of mice with a mixture of the pneumococcal proteins PsaA and PspA is highly protective against nasopharyngeal carriage of Streptococcus pneumoniae. Infect Immun 68, 796-800.

Chapuy-Regaud, S., Ogunniyi, A. D., Diallo, N., Huet, Y., Desnottes, J. F., Paton, J. C., Escaich, S. \& Trombe, M. C. (2003). RegR, a global LacI/GalR family regulator, modulates virulence and competence in Streptococcus pneumoniae. Infect Immun 71, 2615-2625.

Chastanet, A., Prudhomme, M., Claverys, J. P. \& Msadek, T. (2001). Regulation of Streptococcus pneumoniae clp genes and their role in competence development and stress survival. J Bacteriol 183, 72957307.

Chicharro, J. L., Serrano, V., Urena, R., Gutierrez, A. M., Carvajal, A., Fernandez-Hernando, P. \& Lucia, A. (1999). Trace elements and electrolytes in human resting mixed saliva after exercise. Br J Sports Med 33, 204-207.

Dawid, S., Roche, A. M. \& Weiser, J. N. (2007). The blp bacteriocins of Streptococcus pneumoniae mediate intraspecies competition both in vitro and in vivo. Infect Immun 75, 443-451.

de Saizieu, A., Gardes, C., Flint, N., Wagner, C., Kamber, M., Mitchell, T. J., Keck, W., Amrein, K. E. \& Lange, R. (2000). Microarray-based identification of a novel Streptococcus pneumoniae regulon controlled by an autoinduced peptide. J Bacteriol 182, 4696-4703.

Dintilhac, A., Alloing, G., Granadel, C. \& Claverys, J. P. (1997). Competence and virulence of Streptococcus pneumoniae: Adc and PsaA mutants exhibit a requirement for $\mathrm{Zn}$ and $\mathrm{Mn}$ resulting from inactivation of putative ABC metal permeases. Mol Microbiol 25, 727739.

Garcia de la Nava, J., van Hijum, S. \& Trelles, O. (2003). PreP: gene expression data pre-processing. Bioinformatics 19, 2328-2329.

Giammarinaro, P. \& Paton, J. C. (2002). Role of RegM, a homologue of the catabolite repressor protein CcpA, in the virulence of Streptococcus pneumoniae. Infect Immun 70, 5454-5461.

Glover, D. T., Hollingshead, S. K. \& Briles, D. E. (2008). Streptococcus pneumoniae surface protein PcpA elicits protection against lung infection and fatal sepsis. Infect Immun 76, 2767-2776.

Hakenbeck, R., Balmelle, N., Weber, B., Gardes, C., Keck, W. \& de Saizieu, A. (2001). Mosaic genes and mosaic chromosomes: intraand interspecies genomic variation of Streptococcus pneumoniae. Infect Immun 69, 2477-2486.

Hanks, T. S., Liu, M., McClure, M. J., Fukumura, M., Duffy, A. \& Lei, B. (2006). Differential regulation of iron- and manganese-specific MtsABC and heme-specific HtsABC transporters by the metalloregulator MtsR of group A Streptococcus. Infect Immun 74, 5132-5139.

Hava, D. L. \& Camilli, A. (2002). Large-scale identification of serotype 4 Streptococcus pneumoniae virulence factors. Mol Microbiol 45, 13891406.

Hava, D. L., Hemsley, C. J. \& Camilli, A. (2003a). Transcriptional regulation in the Streptococcus pneumoniae rlrA pathogenicity islet by RlrA. J Bacteriol 185, 413-421.

Hava, D. L., LeMieux, J. \& Camilli, A. (2003b). From nose to lung: the regulation behind Streptococcus pneumoniae virulence factors. Mol Microbiol 50, 1103-1110.
Hemsley, C., Joyce, E., Hava, D. L., Kawale, A. \& Camilli, A. (2003). $\mathrm{MgrA}$, an orthologue of Mga, acts as a transcriptional repressor of the genes within the rlrA pathogenicity islet in Streptococcus pneumoniae. J Bacteriol 185, 6640-6647.

Hendriksen, W. T., Silva, N., Bootsma, H. J., Blue, C. E., Paterson, G. K., Kerr, A. R., de Jong, A., Kuipers, O. P., Hermans, P. W. \& Mitchell, T. J. (2007). Regulation of gene expression in Streptococcus pneumoniae by response regulator 09 is strain dependent. J Bacteriol 189, 1382-1389.

Hendriksen, W. T., Bootsma, H. J., Estevao, S., Hoogenboezem, T., de Jong, A., de Groot, R., Kuipers, O. P. \& Hermans, P. W. (2008a). CodY of Streptococcus pneumoniae: link between nutritional gene regulation and colonization. J Bacteriol 190, 590-601.

Hendriksen, W. T., Kloosterman, T. G., Bootsma, H. J., Estevao, S., de Groot, R., Kuipers, O. P. \& Hermans, P. W. (2008b). Site-specific contributions of glutamine-dependent regulator GlnR and GlnRregulated genes to virulence of Streptococcus pneumoniae. Infect Immun 76, 1230-1238.

Ibrahim, Y. M., Kerr, A. R., Silva, N. A. \& Mitchell, T. J. (2005). Contribution of the ATP-dependent protease ClpCP to the autolysis and virulence of Streptococcus pneumoniae. Infect Immun 73, 730-740.

Jakubovics, N. S. \& Jenkinson, H. F. (2001). Out of the iron age: new insights into the critical role of manganese homeostasis in bacteria. Microbiology 147, 1709-1718.

Jakubovics, N. S., Smith, A. W. \& Jenkinson, H. F. (2000). Expression of the virulence-related Sca $\left(\mathrm{Mn}^{2+}\right)$ permease in Streptococcus gordonii is regulated by a diphtheria toxin metallorepressor-like protein ScaR. Mol Microbiol 38, 140-153.

Johnston, J. W., Myers, L. E., Ochs, M. M., Benjamin, W. H., Jr, Briles, D. E. \& Hollingshead, S. K. (2004). Lipoprotein PsaA in virulence of Streptococcus pneumoniae: surface accessibility and role in protection from superoxide. Infect Immun 72, 5858-5867.

Johnston, J. W., Briles, D. E., Myers, L. E. \& Hollingshead, S. K. (2006). $\mathrm{Mn}^{2+}$-dependent regulation of multiple genes in Streptococcus pneumoniae through PsaR and the resultant impact on virulence. Infect Immun 74, 1171-1180.

Kadioglu, A., Gingles, N. A., Grattan, K., Kerr, A., Mitchell, T. J. \& Andrew, P. W. (2000). Host cellular immune response to pneumococcal lung infection in mice. Infect Immun 68, 492-501.

Kerr, A. R., Adrian, P. V., Estevao, S., de Groot, R., Alloing, G., Claverys, J. P., Mitchell, T. J. \& Hermans, P. W. (2004). The Ami-AliA/ AliB permease of Streptococcus pneumoniae is involved in nasopharyngeal colonization but not in invasive disease. Infect Immun 72, 3902-3906.

Kloosterman, T. G., Hendriksen, W. T., Bijlsma, J. J., Bootsma, H. J., van Hijum, S. A., Kok, J., Hermans, P. W. \& Kuipers, O. P. (2006). Regulation of glutamine and glutamate metabolism by GlnR and GlnA in Streptococcus pneumoniae. J Biol Chem 281, 25097-25109.

Kloosterman, T. G., van der Kooi-Pol, M. M., Bijlsma, J. J. \& Kuipers, O. P. (2007). The novel transcriptional regulator SczA mediates protection against $\mathrm{Zn}^{2+}$ stress by activation of the $\mathrm{Zn}^{2+}$-resistance gene czcD in Streptococcus pneumoniae. Mol Microbiol 65, 1049-1063.

Kloosterman, T. G., Witwicki, R. M., van der Kooi-Pol, M. M., Bijlsma, J. J. \& Kuipers, O. P. (2008). Opposite effects of $\mathrm{Mn}^{2+}$ and $\mathrm{Zn}^{2+}$ on the PsaR-mediated expression of the virulence genes $p c p A$, prtA and psaBCA of Streptococcus pneumoniae. J Bacteriol 190, 53825393.

Lanie, J. A., Ng, W. L., Kazmierczak, K. M., Andrzejewski, T. M., Davidsen, T. M., Wayne, K. J., Tettelin, H., Glass, J. I. \& Winkler, M. E. (2007). Genome sequence of Avery's virulent serotype 2 strain D39 of Streptococcus pneumoniae and comparison with that of unencapsulated laboratory strain R6. J Bacteriol 189, 38-51. 
Lau, G. W., Haataja, S., Lonetto, M., Kensit, S. E., Marra, A., Bryant, A. P., McDevitt, D., Morrison, D. A. \& Holden, D. W. (2001). A functional genomic analysis of type 3 Streptococcus pneumoniae virulence. Mol Microbiol 40, 555-571.

Lawrence, M. C., Pilling, P. A., Epa, V. C., Berry, A. M., Ogunniyi, A. D. \& Paton, J. C. (1998). The crystal structure of pneumococcal surface antigen PsaA reveals a metal-binding site and a novel structure for a putative ABC-type binding protein. Structure 6, 15531561.

Long, A. D., Mangalam, H. J., Chan, B. Y., Tolleri, L., Hatfield, G. W. \& Baldi, P. (2001). Improved statistical inference from DNA microarray data using analysis of variance and a Bayesian statistical framework. Analysis of global gene expression in Escherichia coli K12. J Biol Chem 276, 19937-19944.

McAllister, L. J., Tseng, H. J., Ogunniyi, A. D., Jennings, M. P., McEwan, A. G. \& Paton, J. C. (2004). Molecular analysis of the psa permease complex of Streptococcus pneumoniae. Mol Microbiol 53, 889-901.

McCluskey, J., Hinds, J., Husain, S., Witney, A. \& Mitchell, T. J. (2004). A two-component system that controls the expression of pneumococcal surface antigen $\mathrm{A}(\mathrm{PsaA})$ and regulates virulence and resistance to oxidative stress in Streptococcus pneumoniae. Mol Microbiol 51, 1661-1675.

Paik, S., Brown, A., Munro, C. L., Cornelissen, C. N. \& Kitten, T. (2003). The sloABCR operon of Streptococcus mutans encodes an Mn and $\mathrm{Fe}$ transport system required for endocarditis virulence and its Mn-dependent repressor. J Bacteriol 185, 5967-5975.

Paterson, G. K., Blue, C. E. \& Mitchell, T. J. (2006). An operon in Streptococcus pneumoniae containing a putative alkylhydroperoxidase $\mathrm{D}$ homologue contributes to virulence and the response to oxidative stress. Microb Pathog 40, 152-160.
Polissi, A., Pontiggia, A., Feger, G., Altieri, M., Mottl, H., Ferrari, L. \& Simon, D. (1998). Large-scale identification of virulence genes from Streptococcus pneumoniae. Infect Immun 66, 5620-5629.

Romero-Steiner, S., Pilishvili, T., Sampson, J. S., Johnson, S. E., Stinson, A., Carlone, G. M. \& Ades, E. W. (2003). Inhibition of pneumococcal adherence to human nasopharyngeal epithelial cells by anti-PsaA antibodies. Clin Diagn Lab Immunol 10, 246-251.

Romero-Steiner, S., Caba, J., Rajam, G., Langley, T., Floyd, A., Johnson, S. E., Sampson, J. S., Carlone, G. M. \& Ades, E. (2006). Adherence of recombinant pneumococcal surface adhesin A (rPsaA)coated particles to human nasopharyngeal epithelial cells for the evaluation of anti-PsaA functional antibodies. Vaccine 24, 3224-3231.

Sanchez-Beato, A. R., Lopez, R. \& Garcia, J. L. (1998). Molecular characterization of PcpA: a novel choline-binding protein of Streptococcus pneumoniae. FEMS Microbiol Lett 164, 207-214.

Talkington, D. F., Brown, B. G., Tharpe, J. A., Koenig, A. \& Russell, H. (1996). Protection of mice against fatal pneumococcal challenge by immunization with pneumococcal surface adhesin A (PsaA). Microb Pathog 21, 17-22.

Tseng, H. J., McEwan, A. G., Paton, J. C. \& Jennings, M. P. (2002). Virulence of Streptococcus pneumoniae: PsaA mutants are hypersensitive to oxidative stress. Infect Immun 70, 1635-1639.

van Hijum, S. A., Garcia de la Nava, J., Trelles, O., Kok, J. \& Kuipers, O. P. (2003). MicroPreP: a cDNA microarray data pre-processing framework. Appl Bioinformatics 2, 241-244.

Varrot, A., Yamamoto, H., Sekiguchi, J., Thompson, J. \& Davies, G. J. (1999). Crystallization and preliminary X-ray analysis of the 6phospho-alpha-glucosidase from Bacillus subtilis. Acta Crystallogr D Biol Crystallogr 55, 1212-1214.

Edited by: T. Msadek 